\title{
Radical spin interaction in one-dimensional chains of decamethyl $\mathrm{C}_{60}$
}

\author{
Kohei Narita*, Susumu Okada** \\ Graduate School of Pure and Applied Sciences, University of Tsukuba, 1-1-1 Tennodai, \\ Tsukuba, Ibaraki 305-8571, Japan
}

\begin{abstract}
Based on density functional theory, we examine the energetics and electronic structure of chains of decamethyl $\mathrm{C}_{60}$ with two stacking conformations. The decamethyl $\mathrm{C}_{60}$ chains possess various spin configurations arising from the combinations of intramolecular and intermolecular spin interactions. The antiparallel spin arrangement within and between molecules is the ground state, and the intramolecular spin interaction is four times larger than the intermolecular interaction. These decamethyl $\mathrm{C}_{60}$ chains possess small dispersion bands near the Fermi level arising from the $\pi$ states distributed on pentagonal rings, which are well separated from each other because of segmentation by the attached methyl groups.
\end{abstract}

Keywords: Decaorgano $\mathrm{C}_{60}$, Electronic structure, One-dimensional chain, Spin polarization

\section{Introduction}

Fullerenes [1, 2] are used as starting materials to design various derivatives which often possess unusual geometric and electronic properties owing to their hollow-cage structure with nanometer diameter $[3,4]$. For example, it has been pointed out that the nanoscale space inside fullerenes is capable of accommodating foreign atoms and molecules [5], leading to various com-

\footnotetext{
*TEL/FAX: +81-298535600 (ext. 8233)/+81-298535924

**TEL/FAX: +81-298535921/+81-298535924

Email addresses: knarita@comas.frsc.tsukuba.ac.jp (Kohei Narita), sokada@comas.frsc.tsukuba.ac.jp (Susumu Okada)
} 
plexes with interesting physical and chemical properties. Indeed, following the macroscopic production of $\mathrm{C}_{60}$ [6] and other fullerenes $[7,8,9,10,11,12]$, higher fullerenes containing metal atoms (metallofullerenes) were synthesized by laser evaporation of carbon rods containing metal atoms [13, 14, 15, 16]. In addition to the encapsulation of atoms and molecules, chemical modification of the fullerene cages by functional groups leads to various fullerene derivatives with unique geometries and interesting physical properties $[17$, 18, 19, 20, 21, 22]: $\mathrm{C}_{60}$ derivatives with nanometer-scale badminton shuttlecock shape have been synthesized under controlled modifications of $\mathrm{C}_{60}$ by the attachment of five aromatic functional groups $[17,18,19]$. Further selective multiple additions of organic molecules onto pentaorgano $\mathrm{C}_{60}$ results in decaorgano $\mathrm{C}_{60}$, in which the attached organic groups divide the spherical $\pi$ electron system of $\mathrm{C}_{60}$ into two pentagonal $\pi$ rings and a cyclophenanthrene $\pi$ network. Because of this segmentation of spherical $\pi$ networks into one or two pentagonal $\pi$ rings, these fullerene derivatives possess radical spins on the pentagonal $\pi$ rings $[20,23,24]$. In our previous work, the decamethyl $\mathrm{C}_{60}$ was shown to possess various spin states that are tunable by applying an external electric field [24].

Fullerenes and their derivatives are known to form condensed phases in which the molecules act as atom-like constituent units similar to atoms in conventional solids [25]. In the solid phase, the molecules are weakly bound to each other and retain their hollow-cage covalent network. In these condensed phases, because of their structural hierarchy, the electronic properties of the solid phase reflect the characteristic features of each constituent. For example, a face-centered cubic $\mathrm{C}_{60}$ possesses small dispersion band corresponding with its molecular states, leading to the superconductivity by injecting additional electrons into unoccupied $t_{1 u}$ states $[26,27,28,29]$. In our previous calculation, we demonstrated that the stacked pentaorgano $\mathrm{C}_{60}$ possesses an antiferromagnetic spin arrangement in its ground state, rather than the ferromagnetic state [20].

In this work, we aim to investigate the spin states of one-dimensional chains of decamethyl $\mathrm{C}_{60}$ with respect to their inter- and intra-molecular radical spin arrangement using first-principles total-energy calculations based on density functional theory. Our calculations show that an antiparallel spin arrangement between two radical spins, not only in each molecule but also between adjacent molecules, is the most energetically favorable among five possible spin states studied here. Energetics of these spin states revealed that the spin-spin interactions are $48 \mathrm{meV}$ and $11 \mathrm{meV}$ for within and between 
molecules, respectively, indicating that the intramolecular spin interaction dominates the spin-spin interaction in one-dimensional chains of decamethyl $\mathrm{C}_{60}$. Furthermore, the intermolecular radical spin interaction depends on the mutual molecular arrangement in the chains. Our finding clearly shows the possible spin conformations of the decamethyl $\mathrm{C}_{60}$ chain and their energetics, which are important to consider for practical applications employing these molecules in spin electronic devices in the near future.

\section{Calculation methods and structural model}

All calculations were performed using density functional theory [30, 31] as implemented in the Simulation Tool for Atom TEchnology (STATE) package [32]. We use the local spin density approximation to treat the exchangecorrelation energy of interacting electrons [33, 34] to account for the spin degree of freedom of the system and to simulate the weak intermolecular interaction to investigate the radical spin interaction under the equilibrium molecular spacing. Ultrasoft pseudopotentials generated using the Vanderbilt scheme were used to describe the interaction between electrons and ions [35]. The valence wave functions and charge density were expanded by the planewave basis set with cutoff energies of 25 and 225 Ry, respectively.

In this work, to investigate the molecular orientation dependence of the spin interaction in the decamethyl $\mathrm{C}_{60}$ chains, we consider two different intermolecular conformations: the side-to-side conformation in which the highest symmetry molecular axis is normal to the chain direction, and the head-tohead arrangement in which the molecular axis is parallel to the chain. To investigate long-range modulation in the spin arrangements, the unit cell contains two molecules with the same molecular orientation. The atomic structures and intermolecular spacing were optimized until the force acting on each atom was less than $5 \mathrm{mRy} / \AA$. Each molecular chain is separated from its adjacent chains by $12 \AA$-vacuum spacing to exclude the effect arising from the periodic images. Integration over the one-dimensional Brillouin zone was carried out using an equidistance mesh of two k-points which allow us to make qualitative discussion on relative total energy of $\mathrm{C}_{60}$ chain [36]. Under the equilibrium arrangement of decamethyl $\mathrm{C}_{60}$ chains with these molecular conformations, we investigate the total energy of various spin states. 
(a)

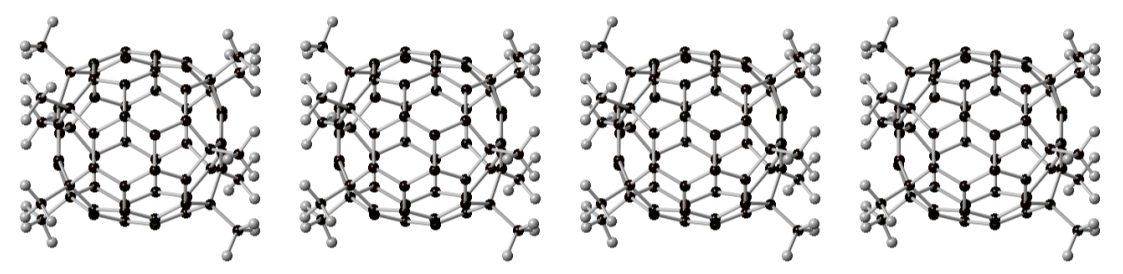

(b)

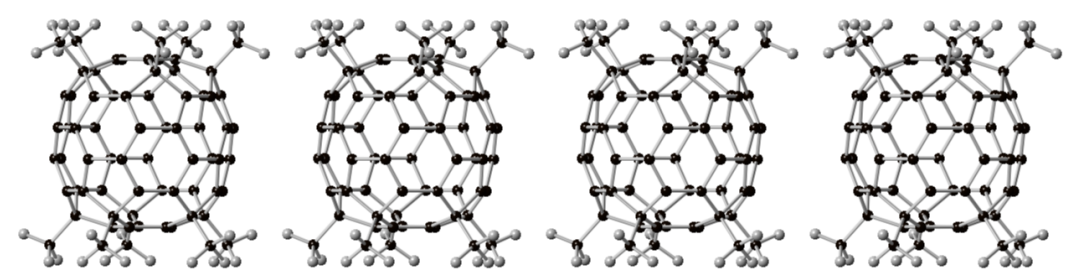

Figure 1: Structural models of decamethyl $\mathrm{C}_{60}$ chains with (a) the head-to-head and (b) side-to-side molecular conformations.

(a)

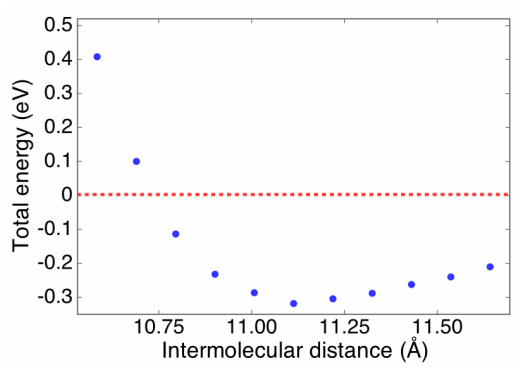

(b)

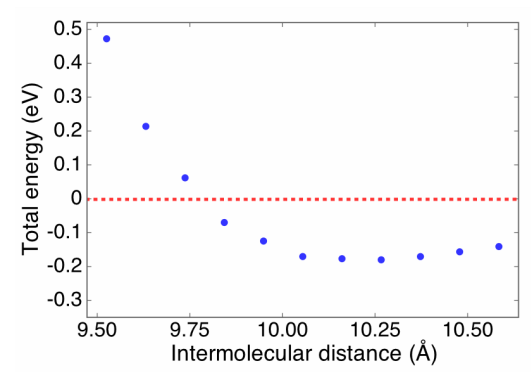

Figure 2: (Color online) Total energies of decamethyl $\mathrm{C}_{60}$ chains with the (a) head-tohead and (b) side-to-side conformations as a function of the intermolecular distance. The energies are relative to the total energy of an isolated decamethyl $\mathrm{C}_{60}$ molecule. 


\section{Results and Discussion}

Figure 2 shows the total energy of the decamethyl $\mathrm{C}_{60}$ chains with the side-to-side and head-to-head molecular conformations as a function of the intermolecular distance, which corresponds to the distance between the center of mass of adjacent molecule. The chains have energy minima at an intermolecular distance of 10.2 and $11.1 \AA$ for the side-to-side and head-to-head conformations, respectively, indicating that the equilibrium intermolecular distance strongly depends on the molecular conformations. The conformation dependence is caused by the steric hindrance of the methyl group attached to the $\mathrm{C}_{60}$. In the case of the side-to-side conformation, the methyl groups are alternately arranged between adjacent decamethyl $\mathrm{C}_{60}$ leading to a small distance which is almost the same at the intermolecular distance of conventional solid $\mathrm{C}_{60}$. In this case, the methyl groups do not affect the intermolecular distance. In contrast, for the head-to-head conformation, the methyl groups act as the steric hindrance between the two molecules, resulting in the remarkable elongation of the intermolecular distance.

At the equilibrium intermolecular distance, the total energies of the decamethyl $\mathrm{C}_{60}$ chains are lower than that of an isolated decamethyl $\mathrm{C}_{60}$ by 188 and $318 \mathrm{meV}$ per molecule for the side-to-side and head-to-head conformations, respectively. Furthermore, the total energy of the chain with intermediate arrangements between the head-to-head and side-to-side arrangements, in which the molecules are tilted by 30 and 60 degree with respect to the chain direction, are 136 and $101 \mathrm{meV}$, indicating that the conformations are less stable than the head-to-head conformation. Thus, the head-to-head conformation is the ground state molecular conformation for one-dimensional chains of decamethyl $\mathrm{C}_{60}$. Note that the intermolecular interaction per molecular coordination number is stronger than that of the solid $\mathrm{C}_{60}$ by about 20 meV. More interestingly, the interaction for the head-to-head conformation is larger than those for the side-to-side and for the pristine $\mathrm{C}_{60}$, although the intermolecular distance is the largest among these three. This fact indicates that the methyl groups attached to $\mathrm{C}_{60}$ play a decisive role in stabilizing the system. Since the methyl groups possesses $\mathrm{CH}$ bonds, interactions between the $\mathrm{CH}$ bonds and $\pi$ systems of the molecule decreases the total energy of the chains. In particular, in the case of the head-to-head conformation, electrostatic interaction between pentagonal rings and $\mathrm{H}$ atoms substantially

stabilizes the chain structure, in which the pentagonal rings and $\mathrm{H}$ atoms tend to be negatively and positively charged, respectively, compared with 
(a)

(AaAa)

(ApAp)
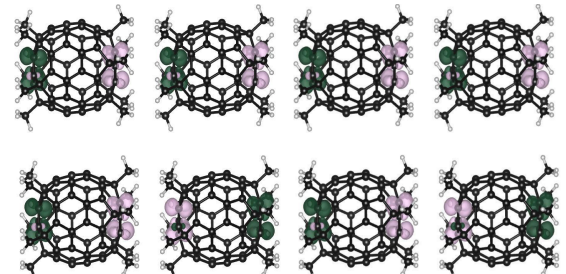

(PpAa)
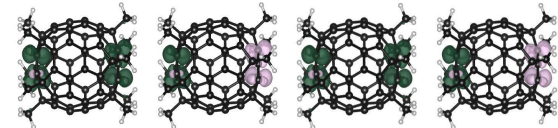

(PaPa)
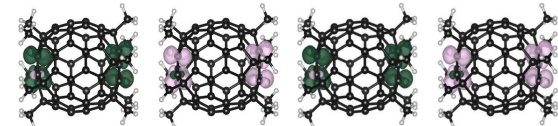

(PpPp)
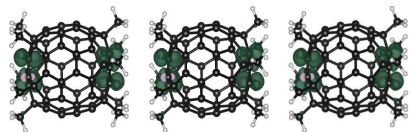

(b)

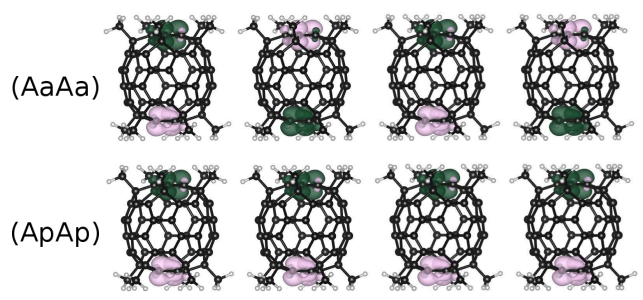

(PpAa)

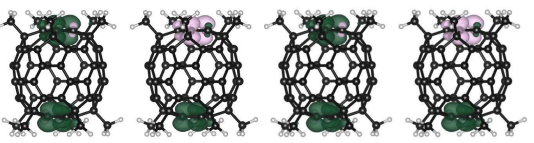

(PaPa)

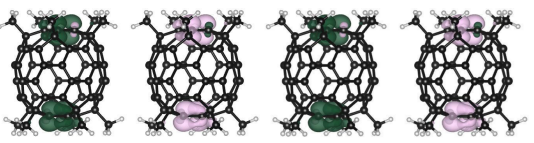

(PpPp)

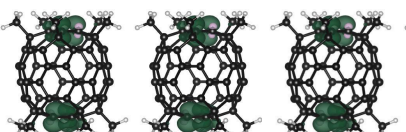

Figure 3: (Color online) Isosurfaces of polarized electron spins of five spin arrangements of decamethyl $\mathrm{C}_{60}$ chains with (a) the head-to-head and (b) side-to-side molecular conformations. Pink and green isosurfaces denote the polarized electrons with majority and minority spin components.

the $\mathrm{C}$ atoms in the hexagonal networks [37]. Therefore, this large binding energy is ascribed to a cooperative effect between the weak intermolecular interactions and $\mathrm{CH} / \pi$ interactions between adjacent decamethyl $\mathrm{C}_{60}[38,39]$.

At the equilibrium intermolecular distance, we investigate the possible spin arrangements of four radical spins distributed on pentagonal rings in the side-to-side and head-to-head conformations. Figure 3 shows possible spin arrangements on decamethyl $\mathrm{C}_{60}$ chains with the side-to-side and head-tohead conformations: We consider antiparallel and parallel spin arrangements between and within the molecules, and find that these five spin arrangements are energetically metastable.

In the case of the head-to-head conformation, among these five spin configurations, the spin state with antiparallel intramolecular and intermolecular spin arrangements (AaAa state) is the ground state in which the polarized electron spins on pentagonal rings are antiferromagnetically aligned along the chain direction. In contrast, the spin state with parallel intramolecular and intermolecular spin arrangements (PpPp state) is the least stable 
state among the five, in which the polarized radical spins are ferromagneticaly aligned through the molecular chain. The remaining three arrangements have stabilities in between the AaAa and PpPp states. These are described as follows: The chains have antiparallel intramolecular and parallel intermolecular spin configurations (ApAp), one of two molecules possesses a parallel spin arrangement with antiparallel and parallel intermolecular spin arrangement (PpAa), and the chains have a parallel intramolecular and an antiparallel intermolecular spin configuration $(\mathrm{PaPa})$. The relative stability of the spin state decreases with increasing amount of parallel spin pairs both within and between molecules. Thus, the radical spins on the decamethyl $\mathrm{C}_{60}$ chains prefer antiparallel over the parallel spin coupling within each molecule and between adjacent molecules.

In the case of the side-to-side conformation, the AaAa is also the ground state. In addition, the ApAp state, in which the spins in each molecule are antiparallelly aligned while the spin between molecules are parallelly aligned, is of the same energy within the numerical error. The result indicates that the intermolecular spin-spin interaction is negligible in decamethyl $\mathrm{C}_{60}$ chains with this molecular conformation. Indeed, the least stable state is also doubly degenerate: both the PaPa and PpPp states have equal energies and are the least stable.

Table 1: Relative total energies per unit cell and the number of polarized electron spins $\left(\Delta N=\int\left[n_{\alpha}(\boldsymbol{r})-n_{\beta}(\boldsymbol{r})\right] d V\right)$ of decamethyl $\mathrm{C}_{60}$ with the side-to-side and head-to-head conformations. Energies are relative to the state with antiparallel intermolecular and intramolecular spin arrangements (AaAa state in Fig. 3).

\begin{tabular}{cccc}
\hline \multirow{2}{*}{ Spin state } & \multirow{2}{*}{$\Delta N$} & \multicolumn{2}{c}{ Energy $(\mathrm{meV})$} \\
\cline { 3 - 4 } & & head-to-head & side-to-side \\
\hline AaAa & 0 & 0 & 0 \\
ApAp & 0 & 22 & 0 \\
PpAa & 2 & 57 & 48 \\
PaPa & 0 & 88 & 96 \\
PpPp & 4 & 118 & 96 \\
\hline
\end{tabular}

To introduce a more quantitative discussion of the energetics of spin states of the decamethyl $\mathrm{C}_{60}$ chains, we present the relative total energies of the spin states in Table 1. By comparing the total energies of the AaAa and ApAp states in the head-to-head conformation, we find that the energy cost 
to flip the antiparallel spin arrangement between molecules into the parallel spin arrangement is $22 \mathrm{meV}$ per unit cell, which corresponds to twice the intramolecular spin-spin interaction energy or the exchange interaction $J_{\text {inter. }}$. As we reported in our previous work, spin-spin interactions within the molecule are $J_{\text {intra }}=42 \mathrm{meV}$. Thus the intermolecular spin interaction is about a fourth of the intramolecular spin-spin interaction, although the distance between adjacent radical spins is smaller by $3 \AA$ than that within the molecule. It is plausible that the large spin-spin interaction within molecules is caused by valence electrons mediating the spin-spin interaction as the case of RKKY interaction [40,41, 42], because of the substantial electron transfer between not only the nearest neighbor but also the next-nearest neighbor atomic sites in $\mathrm{sp}^{2} \mathrm{C}$ network materials. Therefore, the decamethyl $\mathrm{C}_{60}$ chain with the head-to-head conformation is described using a spin chain model containing two exchange interactions $J_{\text {intra }}$ and $J_{\text {inter }}$. By combining the $J_{\text {intra }}$ and $J_{\text {inter }}$, we can quantitatively examine the relative stabilities of all spin states.

In the case of the chain with the side-to-side conformation, we find unusual spin-spin interaction between adjacent molecules. The antiparallel and parallel spin arrangements between molecules are degenerate with each other to within the numerical error because of the large separation between the two radical spin sites $(10 \AA)$. Thus the energetics of the spin states is mainly determined by the individual spin states of each decamethyl $\mathrm{C}_{60}$.

Figure 4 shows the electronic energy bands of the decamethyl $\mathrm{C}_{60}$ chains with the side-to-side and head-to-head conformations in the AaAa ground state spin configuration. In both cases, we find that the electronic states near the Fermi level have dispersion of about $100 \mathrm{meV}$ which is narrower than that of the fcc $\mathrm{C}_{60}(\sim 0.5 \mathrm{eV})$. This small band dispersion is ascribed to the small $\pi-\pi$ overlap between adjacent molecules due to the localized nature of the states near the $\mathrm{E}_{F}$ leading to the intermolecular spin ordering (Fig. 5). In both molecular conformations, methyl groups attached to $\mathrm{C}_{60}$ act as a steric hindrance preventing substantial $\pi-\pi$ overlap between adjacent molecules. Furthermore, in the case of the head-to-head conformation, the segmentation of the $\pi$ network by the methyl groups also causes the small dispersion bands near the Fermi level. Since the state near the Fermi level is localized on the pentagonal rings surrounded by methyl groups, the pentagonal rings are separated from each other by 3 and $7 \AA$ between and within the molecules, respectively.

In the real system, decamethyl $\mathrm{C}_{60}$ is expected to form bulk condensed 


\section{(a)}

(b)

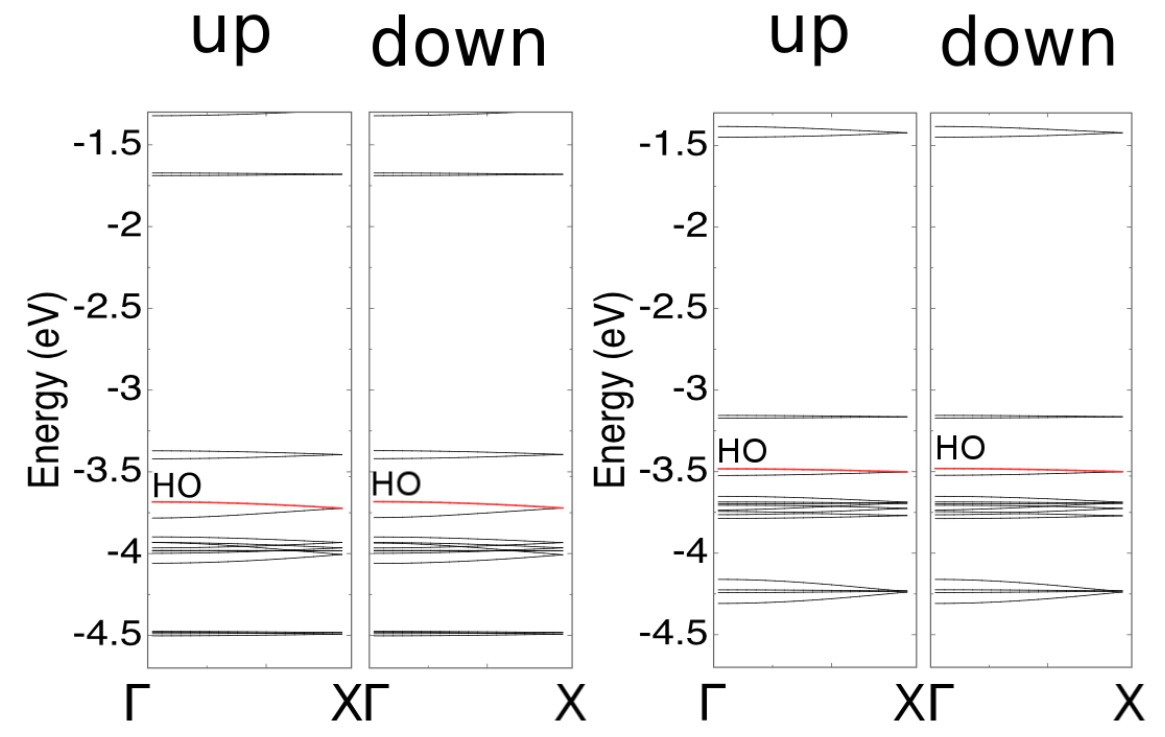

Figure 4: (Color online) Electronic structure of decamethyl $\mathrm{C}_{60}$ chains with (a) head-tohead and (b) side-to-side molecular conformation in the AaAa ground state spin configuration. The energies are relative to the vacuum level. The highest occupied state is denoted by $\mathrm{HO}$. 
(a)

$\mathrm{HO}$

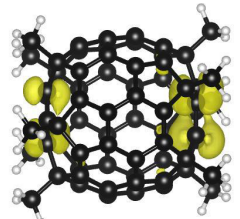

LU

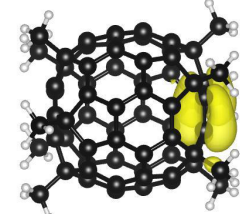

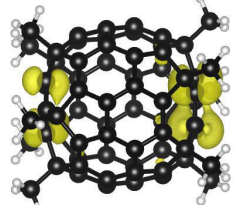

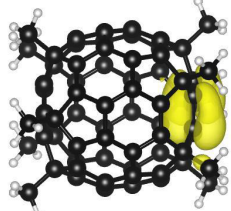

$\mathrm{HO}$

(b)

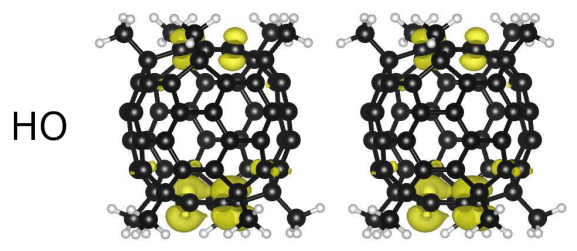

LU

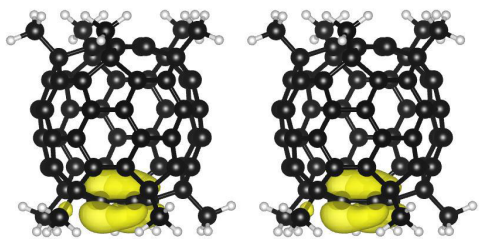

Figure 5: Squared wave function at $\Gamma$ point of the highest occupied and the lowest unoccupied states of decamethyl $\mathrm{C}_{60}$ chains with (a) the head-to-head and (b) side-to-side molecular conformations. In each panel, $\mathrm{HO}$ and $\mathrm{LU}$ denote the wave function of the highest occupied and the lowest unoccupied states, respectively.

phases in which the molecules are three-dimensionally packed in an appropriate manner. In such a solid, because of anisotropic interactions between molecules, the condensed phases may also possess structural anisotropy; it is plausible that the stable condensed phase possesses bundle structures of columns, each of which consists of one-dimensional chains of decamethyl $\mathrm{C}_{60}$ with the head-to-head stacking conformation. Thus, an unusual spin ordering is expected to occur because of the delicate balance among the three antiferromagnetic spin exchange interactions $J$ for the intramolecular, intermolecular, and intercolumnar spin interactions.

\section{Summary}

We studied the energetics and electronic structures of decamethyl $\mathrm{C}_{60}$ chains using density functional theory with the local spin density approximation. Our calculations show that the chain with the head-to-head molecular arrangement is more stable than that with the side-to-side conformation because of the substantial $\mathrm{CH}-\pi$ interaction cooperating with the weak intermolecular interaction. At equilibrium intermolecular distances, we investigate the possible spin configurations for these chains and found five possible 
spin arrangements in the head-to-head conformation, AaAa, ApAp, PpAa, $\mathrm{PaPa}$, and $\mathrm{PpPp}$ where $\mathrm{A}(\mathrm{P})$ and $\mathrm{a}(\mathrm{p})$ denote the antiparallel (parallel) spin coupling within each molecule and between molecules, respectively. Among these spin configurations, the AaAa state is the ground state where all radical spins are antiferromagnetically aligned within and between molecules. On the other hand, a parallel spin coupling has higher total energy than that with the antiparallel spin coupling. Detailed energetics showed that the spin-spin interaction between adjacent molecules is smaller than that within molecule by about a quarter. In contrast, in the case of the side-to-side conformation, because radical spins between adjacent molecules are sufficiently separated, the intermolecular spin interaction is almost negligible compared with the intramolecular interactions. Electronic structures of these decamethyl $\mathrm{C}_{60}$ chains possess small dispersion bands near the Fermi level arising from the $\pi$ states being distributed on pentagonal rings, which are well separated from each other due to segmentation by the methyl groups. These small dispersion bands are the physical origin leading to various spin states on the chains.

\section{Acknowledgments}

This work was supported by CREST, from the Japan Science and Technology Agency, and a Grant-in-Aid for Scientific Research from the Ministry of Education, Culture, Sports, Science and Technology of Japan. A part of the calculations were performed on NEC SX-9 at the Cybermedia Center at Osaka University and on NEC SX-9 at the Institute of Solid State Physics, The University of Tokyo.

[1] H. W. Kroto, J. R. Heath, S. C. O'Brien, R. F. Curl, R. E. Smalley, Nature 318 (1985) 162.

[2] P. W. Fowler, D. E. Manolopoulos, An Atlas of Fullerenes (Oxford University Press, Oxford, 1995).

[3] M. S. Dresselhaus, G. Dresselhaus, P.C. Eklund, Science of Fullerenes and Carbon Nanotubes (Academic Press, San Diego, CA, 1996).

[4] T, Akasaka, F. Wudl, S. Nagase, Chemistry of Nanocarbons (Wiley, West Sussex, 2010)

[5] J.R. Heath, S.C. O'Brien, Q. Zhang, Y. Liu, R.F. Curl, F.K. Tittel, R.E. Smalley, J. Am. Chem. Soc. 107 (1985) 7779. 
[6] W. Krätschmer, L.D. Lamb, K. Fostiropoulous, D.R. Hoffman, Nature 347 (1990) 354.

[7] F. Diederich, R. L. Whetten, C. Thilgen, R. Ettl, I. Chao, M. M. Alvarez, Science 254 (1991) 1768.

[8] K. Kikuchi, N. Nakahara, T. Wakabayashi, S. Suzuki, H. Shiromaru, Y.Miyake, K. Saito, I. Ikemoto, M. Kainosho, Y. Achiba, Nature 357 (1992) 142.

[9] Y. Achiba, K. Kikuchi, Y. Aihara, T. Wakabayashi, Y. Miyake, and M. Kainosho, in "Science and Technology of Fullerene Materials", edited by P. Bernier et. al., (Proc. Mat. Res. Soc. Vol. 359, Pittsburgh, 1995) p. 3 .

[10] M. Saunders, H. A. Jimenez-Vazquez, R. J. Cross, W. E. Billups, C. Gesenberg, A. Gonzalez, W. Luo,R. C. Haddon, F. Diederich, A. Herrmann, J. Am. Chem. Soc. 117(1995) 9305 .

[11] K. Raghavachari, Chem. Phys. Lett. 190 (1992) 397.

[12] D. Bakowies, M. Kolb, W. Thiel, S. Richard, R. Ahlrichs, and M.M. Kappes, Chem. Phys. Lett. 200 (1992) 411.

[13] Y. Chai, T. Guo, C. Jin, R.E. Haufler, L.P. Felipe Chibante, J. Fure, L. Wang, J. Michael Alford, R.E. Smalley, J. Phys. Chem. 95(20) (1991) 7564 .

[14] H. Shinohara, H. Sato, M. Ohkochi, Y. Ando, T. Kodama, T. Shida, T. Kato, Y. Saito, Nature 357 (1992) 52.

[15] C.-R. Wang, T. Kai, T. Tomiyama, T. Yoshida, Y. Kobayashi, E. Nishibori, M. Takata, M. Sakata, H. Shinohara, Angew. Chem. Int. Ed. 40 (2001) 397.

[16] S. Stevenson, G. Rice, T. Glass, K. Harich, F. Cromer, M.R. Jordan, J. Craft, E. Hadju, R. Bible, M.M. Olmstead, K. Maitra, A.J. Fisher, A.L. Balch, H.C. Dorn, Nature 401 (1999) 55.

[17] Y. Matsuo,E. Nakamura, Chem. Rev. 108 (2008) 3016. 
[18] M. Sawamura, K. Kawai, Y. Matsuo, K. Kanie, T. Kato, E. Nakamura, Nature 419 (2003) 702.

[19] Y. Matsuo, A. Muramatsu, R. Hamasaki, N. Mizushita, T. Kato, E. Nakamura, J. Am. Chem. Soc. 126 (2003) 432.

[20] S. Okada, R. Arita, Y. Matsuo, E. Nakamura, A. Oshiyama, H. Aoki, Chem. Phys. Lett. 399 (2004) 157.

[21] E. Nakamura, K. Tahara, Y. Matsuo, M. Sawamura, J. Am. Chem. Soc. 125 (2003) 2834.

[22] Y. Matsuo, E. Nakamura, J. Am. Chem. Soc. 127 (2005) 8457.

[23] H. Nitta, Y. Matsuo, E. Nakamura, S. Okada, Appl. Phys. Express, 6 (2013) 045102.

[24] K. Narita, S. Okada, Chem. Phys. Lett. 614 (2014) 10.

[25] S. Saito, A. Oshiyama, Phys. Rev. Lett. 66 (1991) 2637.

[26] A. F. Hebard, M. J. Rosseinsky, R. C. Haddon, D. W. Murphy, S. H. Glarum, T. T. M. Palstra, A. P. Ramirez, A. R. Kortan, Nature 350 (1991) 600 .

[27] M. J. Rosseinsky, A. P. Ramirez, S. H. Glarum, D. W. Murphy, R. C. Haddon, A. F. Hebard, T. T. M. Palstra, A. R. Kortan, S. M. Zahurak, A. V. Makhija, Phys. Rev. Lett. 66 (1991) 2830.

[28] P. W. Stephens, L. Mihaly, P. L. Lee, R. L. Whetten, S.-M Huang, R. Kaner, F. Deiderich, K. Holczer, Nature 351 (1991) 632.

[29] K. Tanigaki, T. W. Ebbesen, S. Saito, J. Mizuki, J. S. Tsai, Y. Kubo, S. Kuroshima, Nature 352 (1991) 222.

[30] P. Hohenberg, W. Kohn Phys. Rev. 136 (1964) B864.

[31] W. Kohn, L. J. Sham, Phys. Rev. 140 (1965) A1133.

[32] Y. Morikawa, K. Iwata, K. Terakura, Appl. Surf. Sci. 169-170 (2000) 11.

[33] J. P. Perdew, A. Zunger, Phys. Rev. B 23 (1981) 5048. 
[34] D. M. Ceperley, B. J. Alder, Phys. Rev. Lett. 45 (1980) 566.

[35] D. Vanderbilt, Phys. Rev. B 41 (1990) 7892.

[36] S. Okada, S. Saito, A. Oshiyama, Phys. Rev. Lett. 86 (2001) 3835.

[37] M. Otani, S. Okada, Y. Okamoto, Phys. Rev. B 80 (2009) 153413.

[38] J. Ran, M. W. Wong, J. Phys. Chem. A 110 (2006) 9702.

[39] K. Kamiya, S. Okada, Jpn. J. Appl. Phys. 52 (2013) 04CN07.

[40] M.A. Ruderman, C. Kittel, Phys. Rev. 96, (1954) 99.

[41] T. Kasuya, Prog. Theor. Phys. 16, (1956) 45.

[42] K. Yosida, Phys. Rev. 106, (1957) 893. 

${ }^{*}$ Graphical Abstract

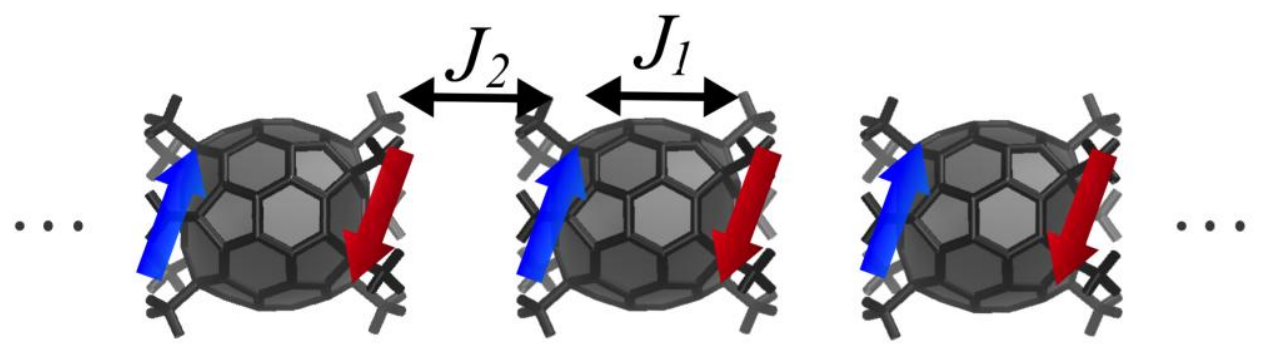

